infection among men who have sex with men over 50 years in Shenzhen, China.

Methods Snowball sampling and respondent driven sampling were used to recruit MSM from 2009 to 2015 in Shenzhen, China. Questionnaire-based interviews were conducted on a one-on-one basis to collect data of socio-demographic information, HIV testing history, history of blood donation in recent two years, self-identified sexual orientation, role in homosexual behaviour, ever being money boy(MB) and clients of $\mathrm{MB}$. $5 \mathrm{ml}$ blood samples were taken and tested for treponema pallidum and HIV antibodies.

Results Among the total of 5221 MSM recruited, 186 $(3.56 \%)$ cases were aged 50 years and older. MSM aged 50 years and older were more likely to get married, have high school or below level of education, have monthly income less than 3000 Yuan, work in service industry or be jobless. Most of them never used condoms for every act with female sexual partners. About one-third had more than 5 anal sexual partners in recent six months. They had higher rate of never using condoms when having anal sex compared with MSM aged less than 50 years. The rate of syphilis, HIV infection and syphilis-HIV co-infection among MSM aged 50 and older were $1.8917,1.7387,2.0365$ times as high as that among MSM aged less 50 years, respectively.

Conclusion MSM aged 50 and older had higher prevalence of syphilis and HIV and acted as bridge population to transmit syphilis/HIV. It is necessary to carry intervention measures targeted to them.

\section{P3.32 ADOLESCENTS INFECTED BY SYPHILIS - A RETROSPECTIVE COHORT IN A REFERENCE SERVICE}

${ }^{1}$ Souza CS, ${ }^{2}$ Xavier-Souza E, 'Lessa KP, ${ }^{1}$ Trindade R, ${ }^{2} \mathrm{MS}$ Timbó, ${ }^{1,3} \mathrm{AG}$ Travassos. ${ }^{1}$ Universidade do Estado da Bahia, Brazil; ${ }^{2}$ Universidade Federal da Bahia, Brazil; ${ }^{3}$ Centro Estadual Especializado em Diagnóstico, Assistência e Pesquisa (CEDAP), Brazil

10.1136/sextrans-2017-053264.269

Introduction Syphilis persists as a major and ascendant health issue. However, the impact of this disease during the adolescence, a period of behavioural, sexual and psychologic vulnerabilities, is still underexplored. We aimed to evaluate the follow-up of syphilis infected adolescents attended at a reference service of sexually transmitted infections (STI).

Methods A retrospective cohort study using data from medical records of adolescents (10 to 19 years old) with diagnosis of syphilis attended at the states STI reference centre, from January to August 2012.

Results Among 776 adolescents attending the service, 58 had a diagnosis of syphilis $(7.47 \%)$. The majority was male $(51.7 \%)$, mean age was $16.8( \pm 1.35)$ years, $92.3 \%$ attended school, and $58.1 \%$ had $\leq 8$ years of schooling; $39.4 \%$ referred drinking alcohol and $39.5 \%$ drug use. Among the adolescents, $98.3 \%$ had already initiated sexual life, with the mean age of sexual debut of $14.2( \pm 1.48)$ years, with an average of 2.33 $( \pm 2.55)$ lifetime sexual partners. About $14.0 \%$ declared to be homosexual, $85.4 \%$ referred irregular condom use, $22.2 \%$ of the girls were pregnant, with mean age at first gestation of $16.0( \pm 1.09)$ years. A parcel of $4.8 \%$ of the adolescents were HIV-infected, $31.0 \%$ reported a previous STI $(p=0.02$, OR 2.68, 95\% CI 1.16-6.17), and 52.3\% had another current STI. The mean number of medical visits was $2.33,22.8 \%$ had primary syphilis, $17.5 \%$ had the secondary phase of the disease and $59.6 \%$ had latent or late syphilis. The social and demographic variables were not statistically different among the adolescents in the different stages of syphilis. The genital ulcer complaint was related to the diagnosis of primary or secondary syphilis $(\mathrm{p}=0.01$, OR $8.53,95 \%$ CI 1.61-45.1). $91.4 \%$ of adolescents received treatment for syphilis and $56.6 \%$ performed a cure control.

Conclusion The high prevalence of other STIs associated with syphilis in adolescents demonstrates the limited knowledge of this population to care and prevention strategies, and remains a challenge for specialised services in the diagnosis and treatment of STI/HIV.

\section{P3.33 SURVEILLANCE OF SYPHILIS IN THE STATE OF SÃO PAULO, BRAZIL}

Carla Gianna Luppi, Valdir Monteiro Pinto, Carmen Silvia Bruniera Domingues, Solange Eduardo Chabu Gomes, Roberto José Carvalho da Silva, Angela Tayra, Mariza Vono Tancredi. Centro de Referência e Treinamento em DST/Aids- Programa Estadual de DST/Aids- Secretaria de Estado da Saúde de São Paulo, Brazil

\subsection{6/sextrans-2017-053264.270}

Introduction In Brazil, has been mandatory to report congenital syphilis (1986), syphilis in pregnancy (2005) and acquired syphilis (adults) (2010) to the national surveillance system. Until June 2016, 227663 cases of acquired syphilis were reported in Brazil, the state of São Paulo accounted for 44\%. The aim of this study was to describe the trends observed from 2011 to 2015 of reported cases of acquired syphilis, syphilis in pregnancy and congenital syphilis in the state of São Paulo.

Methods An ecological study of historical series was conducted, assessing data of reported cases from 2011 to 2015 in the State of São Paulo, Brazil. The goodness of fit via $r^{2}$ and $\mathrm{p}<0.05$ were used to determine which models were most appropriate.

Results In the period 12312 cases of congenital syphilis (CS), 25399 cases of syphilis in pregnancy and 93937 cases of acquired syphilis were notified. There was an increase number of cases (2011 and 2015): CS (1,517 to 3,437 - increased 2.3 times); syphilis in pregnancy $(3,205$ to 6,956 - increased 2.2 times); acquired syphilis $(10,841$ to 25,987 - increased 2.4 times). There was a trend of increase for the three reported diseases with an annual mean increase of 3894 cases/year $\left(\mathrm{r}^{2}=0.9938\right)$ for acquired syphilis, 998 cases/year $\left(\mathrm{r}^{2}=0.9849\right)$ for syphilis in pregnancy and 490 cases/years $\left(r^{2}=0.9969\right)$ for CS. Analysing acquired syphilis by gender, there was a trend of 2528 cases/year increase in men (higher in the age from 20 to 24 years - 488 cases/year) and 1366 cases/ear increase in women (higher in the age from 20 to 24 years - 264 cases/year). Among reported cases of syphilis in pregnancy, the highest increase was in the age of 20 to 24 years with 350 cases/year.

Conclusion The increase in reported syphilis cases could be explained by the reduction of underreporting; increase in early diagnosis of pregnant women; increase in cases of specific populations with higher frequency of young men. It is urgent to implement preventive and control actions towards young population, which seems to be disproportionally affected. 